$\frac{\text { DE }}{G}$

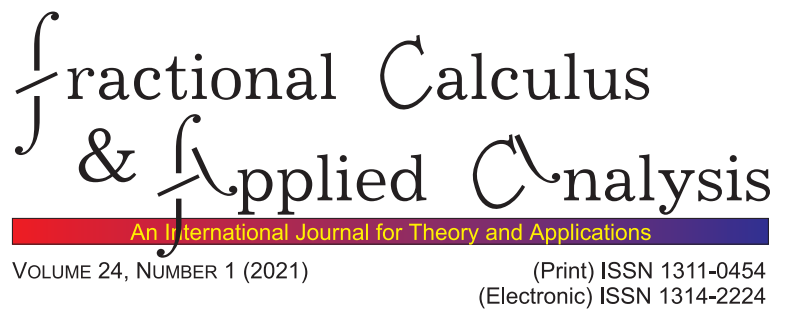

RESEARCH PAPER

\title{
A FRACTIONAL GENERALIZATION OF THE DIRICHLET DISTRIBUTION AND RELATED DISTRIBUTIONS
}

\author{
Elvira Di Nardo ${ }^{1}$, Federico Polito ${ }^{1}$, Enrico Scalas ${ }^{2}$
}

\begin{abstract}
This paper is devoted to a fractional generalization of the Dirichlet distribution. The form of the multivariate distribution is derived assuming that the $n$ partitions of the interval $\left[0, W_{n}\right]$ are independent and identically distributed random variables following the generalized Mittag-Leffler distribution. The expected value and variance of the one-dimensional marginal are derived as well as the form of its probability density function. A related generalized Dirichlet distribution is studied that provides a reasonable approximation for some values of the parameters. The relation between this distribution and other generalizations of the Dirichlet distribution is discussed. Monte Carlo simulations of the one-dimensional marginals for both distributions are presented.
\end{abstract}

MSC 2010: 60E05; 33E12, 60G22

Key Words and Phrases: fractional Dirichlet distribution; generalized Dirichlet distribution; three-parameter Mittag-Leffler functions; fractional Poisson process; wealth distribution; power-law tails

\section{Introduction}

Let us consider a finite sequence of $n$ positive random variables $Z_{1}, \ldots, Z_{n}$. For instance, these variables can represent the wealth of $n$ economic agents if indebtedness is not allowed. Let us denote the sum as $W_{n}=Z_{1}+\ldots+Z_{n}$. In the wealth interpretation this is the total wealth. If we define the

(C) 2021 Diogenes Co., Sofia

pp. 112-136, DOI: 10.1515/fca-2021-0006

DE GRUYTER 
A FRACTIONAL GENERALIZATION OF THE DIRICHLET... 113

wealth fraction of the $i$-th agent as $Q_{i}=Z_{i} / W_{n}$, we get a partition of the interval $[0,1]$ represented by the sequence $\mathbf{Q}=\left(Q_{1}, \ldots, Q_{n}\right)$ such that $Q_{1}+\ldots+Q_{n}=1$ almost surely. We are particularly interested in multivariate distributions for the sequence $\mathbf{Q}$ whose one-dimensional marginals have heavy tails. If we further assume that the random variables $Z_{1}, \ldots, Z_{n}$ are independent and identically distributed, there is a nice and immediate relationship with point processes of renewal type. In this case, the variables $Z_{i}$ can be interpreted as inter-event intervals and the partial sums $W_{k}=\sum_{i=1}^{k} Z_{i}$, with $k \leq n$, are the epochs of the events.

In order to clarify the relationship, we start by recalling some basic facts on the time-fractional Poisson process as we are going to use and generalize it in the next section. From [1, 14] we know that the time-fractional Poisson process $N^{\nu}=\left(N^{\nu}(t)\right)_{t \geq 0}, \nu \in(0,1]$, can be defined as a renewal process with independent and identically distributed inter-event waiting times $\mathcal{T}_{j}$, $j \in \mathbb{N}^{*}=\{1,2, \ldots\}$, with probability density function (pdf)

$$
\mathbb{P}\left(\mathcal{T}_{j} \in \mathrm{d} t\right)=\lambda t^{\nu-1} E_{\nu, \nu}\left(-\lambda t^{\nu}\right) \mathrm{d} t, \quad \lambda>0, t>0,
$$

where

$$
E_{\alpha, \beta}(z)=\sum_{r=0}^{\infty} \frac{z^{r}}{\Gamma(\alpha r+\beta)}, \quad z, \alpha, \beta \in \mathbb{C}, \Re(\alpha)>0,
$$

is the two-parameter Mittag-Leffler function. Note that for $\nu=1$, the waiting times $\mathcal{T}_{j}$ are exponentially distributed and $N^{1}$ is the homogeneous Poisson process. Moreover, the Laplace transform of the pdf (1.1) takes a very compact form. Indeed, we have

$$
\int_{0}^{\infty} e^{-z t} \mathbb{P}\left(\mathcal{T}_{j} \in \mathrm{d} t\right)=\frac{\lambda}{\lambda+z^{\nu}}, \quad z>0
$$

Let us now indicate with $T_{k}, k \in \mathbb{N}^{*}$, the random occurrence time of the $k$-th event of the stream of events defining $N^{\nu}$. From the renewal structure of $N^{\nu}$ we readily obtain that the Laplace transform of $T_{k}$ reads

$$
\int_{0}^{\infty} e^{-z t} \mathbb{P}\left(T_{k} \in \mathrm{d} t\right)=\left(\frac{\lambda}{\lambda+z^{\nu}}\right)^{k}, \quad z>0
$$

which in turn corresponds to the Laplace transform of a function involving the three-parameter Mittag-Leffler function (also known as the Prabhakar function - see [7]). In particular, the three-parameter Mittag-Leffler function is defined as

$$
E_{\alpha, \beta}^{\delta}(z)=\sum_{r=0}^{\infty} \frac{z^{r}}{\Gamma(\alpha r+\beta)} \frac{\Gamma(\delta+r)}{r ! \Gamma(\delta)}, \quad z, \alpha, \beta, \delta \in \mathbb{C}, \Re(\alpha)>0,
$$


and we know by direct calculation that (see e.g. [15], formula (2.3.24))

$$
\int_{0}^{\infty} t^{\beta-1} e^{-z t} E_{\alpha, \beta}^{\delta}\left(\zeta t^{\alpha}\right) \mathrm{d} t=z^{-\beta}\left(1-\zeta z^{-\beta}\right)^{-\delta}
$$

where $\Re(\alpha)>0, \Re(\beta)>0, \Re(z)>0, z>|\zeta|^{1 / \Re(\alpha)}$. Using (1.6), we obtain

$$
\mathbb{P}\left(T_{k} \in \mathrm{d} t\right)=\lambda^{k} t^{\nu k-1} E_{\nu, \nu k}^{k}\left(-\lambda t^{\nu}\right) \mathrm{d} t, \quad \lambda>0, t>0, \nu \in(0,1], k \in \mathbb{N}^{*} .
$$

REMARK 1.1. Note that, for $\nu=1$, the above density reduces to that of an $\operatorname{Erlang}(\lambda, k)$ distributed random variable. This can be seen by simply noticing that

$$
\mathbb{P}\left(T_{k} \in \mathrm{d} t\right)=\mathrm{d} t \lambda^{k} t^{k-1} \sum_{r=0}^{\infty} \frac{(-\lambda t)^{r} \Gamma(r+k)}{\Gamma(r+k) \Gamma(k) r !}=\mathrm{d} t \frac{\lambda^{k} t^{k-1} e^{-\lambda t}}{(k-1) !}, \quad k \in \mathbb{N}^{*} .
$$

REMARK 1.2. The Erlang $(\lambda, k)$ distribution is a special case of the $\operatorname{Gamma}(a, c)$ distribution. Consider a sequence $Z_{1}, \ldots, Z_{n}$ of independent random variables each following a Gamma distribution of parameter $\left(a_{1}, c\right), \ldots,\left(a_{n}, c\right)$. It is well known that their sum $W_{n}$ is still a Gamma of parameter $\left(a_{1}+\ldots+a_{n}, c\right)$. Then the sequence of fractions $Q_{1}, \ldots, Q_{n}$ has a joint $(N-1)$-dimensional Dirichlet distribution of parameters $a_{1}, \ldots, a_{n}$ with density

$$
f_{\mathbf{Q}}\left(q_{1}, \ldots, q_{n-1}\right)=\frac{\Gamma\left(a_{1}+\ldots+a_{n}\right)}{\Gamma\left(a_{1}\right) \cdot \ldots \cdot \Gamma\left(a_{n}\right)} q_{1}^{a_{1}-1} \ldots\left(1-\sum_{i=1}^{n-1} q_{i}\right)^{a_{n}-1}
$$

with $q_{1}+\ldots+q_{n}=1$ and is independent of $W_{n}$.

The proof of the results in Remark 1.2 can be found in several textbooks and lecture notes (see e.g. [2, Lemma 1.5).

REMARK 1.3. The random variables $\mathcal{T}_{j}$ have the following asymptotic behaviour for $t \rightarrow \infty$ [8]:

$$
\mathbb{P}\left(\mathcal{T}_{1}>t\right) \sim \frac{\sin (\nu \pi)}{\pi} \frac{\Gamma(\nu)}{t^{\nu}}, \quad t \gg 1 ;
$$

therefore, their sums $T_{k}$ belong to the basin of attraction of the $\nu$-stable subordinator.

REMARK 1.4. The distributions considered in the present paper belong to the class of distributions on the simplex discussed in [5] (see (2.2) below and [3]). 


\section{A FRACTIONAL GENERALIZATION OF THE DIRICHLET... 115}

This paper contains the following material. Section 2 concerns the definition and properties of the fractional Dirichlet distribution. Section 3 mirrors Section 2 and is devoted to the generalized Dirichlet distribution. Section 4 explains how to simulate the fractional Dirichlet distribution and presents the results of Monte Carlo simulations in order to illustrate the relation between the fractional Dirichlet distribution and the generalized Dirichlet distribution.

\section{Construction of the fractional Dirichlet distribution}

Based on Remark 1.1 and Remark 1.2, we now define a generalization of the Gamma distribution and we immediately present a fractional generalization of the Dirichlet distribution.

Definition 2.1 (Fractional Gamma distribution). Let $X$ be a positive real valued random variable with distribution

$$
\mu(\mathrm{d} x)=\mathbb{P}(X \in \mathrm{d} x)=\lambda^{\beta} x^{\nu \beta-1} E_{\nu, \nu \beta}^{\beta}\left(-\lambda x^{\nu}\right) \mathrm{d} x,
$$

where $\lambda>0, x>0, \beta>0, \nu \in(0,1]$. Then $X$ is said to be distributed as a fractional Gamma of parameters $\lambda, \beta, \nu$ (we write $X \sim F G(\lambda, \beta, \nu)$ ) (see [19]; for applications to renewal processes see [4, 16, 17, 18]).

REMARK 2.1. The Laplace transform of $\mu$ reads

$$
\int_{0}^{\infty} e^{-z x} \mu(\mathrm{d} x)=\left(\frac{\lambda}{\lambda+z^{\nu}}\right)^{\beta}, \quad z>0 .
$$

By means of (2.1), we will construct a generalization of the Dirichlet distribution. We consider $n$ independent random variables $Z_{i}, i=1, \ldots, n$, distributed as fractional Gamma random variables of parameters $\left(1, \beta_{i}, \nu\right)$, $\nu \in(0,1], \beta_{i}>0, i=1, \ldots, n$, respectively. Furthermore, define the sum $W=Z_{1}+\ldots+Z_{n}$, set $Q_{i}=Z_{i} / W, i=1, \ldots, n$, and consider the transformation

$$
\left(Z_{1}, \ldots, Z_{n}\right) \longrightarrow\left(W Q_{1}, \ldots, W Q_{n-1}, W\left(1-\sum_{i=1}^{n-1} Q_{i}\right)\right) .
$$

vskip-2pt Note that, from (2.2), the distribution of $W$ is fractional Gamma as well, i.e. $W \sim F G(1, \bar{\beta}, \nu)$, where $\bar{\beta}=\sum_{i=1}^{n} \beta_{i}$. The joint pdf of the vector $(W, \boldsymbol{Q})=\left(W, Q_{1}, \ldots, Q_{n-1}\right)$ reads

$$
f_{(W, Q)}\left(y, q_{1}, \ldots, q_{n-1}\right)
$$




$$
\begin{aligned}
= & {\left[\prod_{i=1}^{n-1}\left(y q_{i}\right)^{\nu \beta_{i}-1} E_{\nu, \nu \beta_{i}}^{\beta_{i}}\left(-\left(y q_{i}\right)^{\nu}\right)\right]\left[y\left(1-\sum_{i=1}^{n-1} q_{i}\right)\right]^{\nu \beta_{n}-1} } \\
& \times E_{\nu, \nu \beta_{n}}^{\beta_{n}}\left(-\left(y-y \sum_{i=1}^{n-1} q_{i}\right)^{\nu}\right) y^{n-1} \\
= & \left(\prod_{i=1}^{n-1} y^{\nu \beta_{i}-1}\right) y^{\nu \beta_{n}-1} y^{n-1}\left[\prod_{i=1}^{n-1} q_{i}^{\nu \beta_{i}-1} E_{\nu, \nu \beta_{i}}^{\beta_{i}}\left(-\left(y q_{i}\right)^{\nu}\right)\right] \\
& \times\left(1-\sum_{i=1}^{n-1} q_{i}\right)^{\nu \beta_{n}-1} E_{\nu, \nu \beta_{n}}^{\beta_{n}}\left(-\left(y-y \sum_{i=1}^{n-1} q_{i}\right)^{\nu}\right) \\
= & y^{\nu \bar{\beta}-1}\left[\prod_{i=1}^{n-1} q_{i}^{\nu \beta_{i}-1} E_{\nu, \nu \beta_{i}}^{\beta_{i}}\left(-\left(y q_{i}\right)^{\nu}\right)\right]\left(1-\sum_{i=1}^{n-1} q_{i}\right)^{\nu \beta_{n}-1} \\
& \times E_{\nu, \nu \beta_{n}}^{\beta_{n}}\left(-\left(y-y \sum_{i=1}^{n-1} q_{i}\right)^{\nu}\right) .
\end{aligned}
$$

The joint pdf of $\boldsymbol{Q}=\left(Q_{1}, \ldots, Q_{n-1}\right)$ is then obtained by marginalization. Hence,

$$
\begin{aligned}
& f_{\boldsymbol{Q}}\left(q_{1}, \ldots, q_{n-1}\right)=\left(\prod_{i=1}^{n-1} q_{i}^{\nu \beta_{i}-1}\right)\left(1-\sum_{i=1}^{n-1} q_{i}\right)^{\nu \beta_{n}-1} \\
& \quad \times \int_{0}^{\infty} y^{\nu \bar{\beta}-1} \prod_{i=1}^{n-1} E_{\nu, \nu \beta_{i}}^{\beta_{i}}\left(-\left(y q_{i}\right)^{\nu}\right) E_{\nu, \nu \beta_{n}}^{\beta_{n}}\left(-\left(y-y \sum_{i=1}^{n-1} q_{i}\right)^{\nu}\right) \mathrm{d} y .
\end{aligned}
$$

REMARK 2.2. On the $n$-dimensional simplex $\Delta_{n}$ the probability density of the random vector $\left(Q_{1}, \ldots, Q_{n}\right)$, where $\sum_{n} Q_{n}=1$ a.s., writes

$$
\begin{aligned}
& \mathbb{P}\left(\left(Q_{1}, \ldots, Q_{n}\right) \in \mathrm{d}\left(q_{1}, \ldots, q_{n}\right)\right) \\
& =\prod_{i=1}^{n} q_{i}^{\nu \beta_{i}-1} \int_{0}^{\infty} y^{\nu \bar{\beta}-1} \prod_{i=1}^{n} E_{\nu, \nu \beta_{i}}^{\beta_{i}}\left(-\left(y q_{i}\right)^{\nu}\right) \mathrm{d} y .
\end{aligned}
$$

Notice that for $\nu=1$ the integral in the rhs of (2.6) can be easily solved and the $\operatorname{Dirichlet}\left(\boldsymbol{\beta}=\left(\beta_{1}, \ldots, \beta_{n}\right)\right)$ is obtained. In this case $\left(Q_{1}, \ldots, Q_{n}\right)$ is uniformly distributed on $\Delta_{n}$ for $\beta_{i}=1, i \in \mathbb{N}^{*}$.

If $\nu \in(0,1)$ with $\beta_{i}=1$, we have 
A FRACTIONAL GENERALIZATION OF THE DIRICHLET... 117

$$
\mathbb{P}\left(\left(Q_{1}, \ldots, Q_{n}\right) \in \mathrm{d}\left(q_{1}, \ldots, q_{n}\right)\right)=\prod_{i=1}^{n} q_{i}^{\nu-1} \int_{0}^{\infty} y^{\nu n-1} \prod_{i=1}^{n} E_{\nu, \nu}\left(-\left(y q_{i}\right)^{\nu}\right) \mathrm{d} y,
$$

which is symmetric but not uniform.

If we let instead $\beta_{i}=1 / \nu$ (again symmetric), we obtain

$$
\mathbb{P}\left(\left(Q_{1}, \ldots, Q_{n}\right) \in \mathrm{d}\left(q_{1}, \ldots, q_{n}\right)\right)=\int_{0}^{\infty} y^{n-1} \prod_{i=1}^{n} E_{\nu, 1}^{1 / \nu}\left(-\left(y q_{i}\right)^{\nu}\right) \mathrm{d} y .
$$

2.1. Properties. The derivation of the marginal moments can be done explicitly using the formulas in Section 2.2 of [5].

Proposition 2.1. Let $\boldsymbol{Q}=\left(Q_{1}, \ldots, Q_{n-1}\right)$ be a random vector distributed with pdf (2.5). For each $j=1, \ldots, n-1$, we have,

$$
\begin{aligned}
& \mathbb{E} Q_{j}=\frac{\beta_{j}}{\bar{\beta}}, \\
& \operatorname{Var} Q_{j}=\frac{\beta_{j}\left(\bar{\beta}-\beta_{j}\right)}{\bar{\beta}^{2}(\bar{\beta}+1)}(1+\bar{\beta}(1-\nu)) .
\end{aligned}
$$

P r o o f. By Proposition 2 of [5] we have

$$
\begin{aligned}
\mathbb{E} Q_{j} & =-\int_{0}^{\infty}\left(\frac{\mathrm{d}}{\mathrm{d} z}\left(\frac{1}{1+z^{\nu}}\right)^{\beta_{j}}\right)\left(\frac{1}{1+z^{\nu}}\right)^{\bar{\beta}-\beta_{j}} \mathrm{~d} z \\
& =\int_{0}^{\infty} \frac{\beta_{j} \nu z^{\nu-1}}{1+z^{\nu}}\left(1+z^{\nu}\right)^{-\bar{\beta}} \mathrm{d} z \\
& =\beta_{j} \int_{0}^{\infty} \frac{\mathrm{d} w}{(1+w)^{\beta \overline{+} 1}}=\frac{\beta_{j}}{\bar{\beta}} .
\end{aligned}
$$

Similarly, the second moment writes

$$
\begin{aligned}
\mathbb{E} Q_{j}^{2} & =\int_{0}^{\infty} z\left(\frac{\mathrm{d}^{2}}{\mathrm{~d} z^{2}}\left(\frac{1}{1+z^{\nu}}\right)^{\beta_{j}}\right)\left(\frac{1}{1+z^{\nu}}\right)^{\bar{\beta}-\beta_{j}} \mathrm{~d} z \\
& =\int_{0}^{\infty}\left[\frac{\nu^{2} z^{2 \nu-2} \beta_{j}\left(\beta_{j}+1\right)}{\left(1+z^{\nu}\right)^{\beta_{j}+2}}-\frac{\beta_{j} \nu(\nu-1) z^{\nu-2}}{\left(1+z^{\nu}\right)^{\beta_{j}+1}}\right]\left(1+z^{\nu}\right)^{-\bar{\beta}+\beta_{j}} \mathrm{~d} z \\
& z^{\nu}=w \\
& =\beta_{j}\left(\beta_{+} 1\right) \int_{0}^{\infty} \frac{w}{(1+w)^{\bar{\beta}+2}} \mathrm{~d} w+(1-\nu) \beta_{j} \int_{0}^{\infty} \frac{\mathrm{d} w}{(1+w)^{\bar{\beta}+1}} \\
& =\nu \frac{\beta_{j}\left(\beta_{j}+1\right)}{\bar{\beta}(\bar{\beta}+1)}+(1-\nu) \frac{\beta_{j}}{\bar{\beta}},
\end{aligned}
$$


and hence after some computation

$$
\operatorname{Var} Q_{j}=\frac{\beta_{j}\left(\bar{\beta}-\beta_{j}\right)}{\bar{\beta}^{2}(\bar{\beta}+1)}(1+\bar{\beta}(1-\nu)) .
$$

REMARK 2.3. Notice that the first factor of the variance (2.10) is in fact the variance of a one-dimensional marginal of a $\operatorname{Dirichlet}(\boldsymbol{\beta})$ distribution. It follows that the marginals are overdispersed with respect to those of a $\operatorname{Dirichlet}(\boldsymbol{\beta})$ distribution.

We now proceed by analyzing the aggregation property and therefore the marginal distributions.

Proposition 2.2 (Aggregation property). Consider the pdf defined in equation (2.5) and the random variable $\mathcal{Q}=\sum_{j=1}^{k} Q_{i_{j}}$ where $1<k<n$ and $i_{j}$ denotes any permutation of the indices. Then the random variable $\mathcal{Z}=W \mathcal{Q}$ has pdf (2.1) with $\beta=\sum_{j=1}^{k} \beta_{i_{j}}$.

P r o o f. The proof is immediate considering that $\mathcal{Q}$ comes from the sum of i.i.d. positive random variables each one with Laplace transform given by (2.2) and then divided by $W$. Therefore $\mathcal{Z}=W \mathcal{Q}$ has pdf given by (2.1) with $\beta=\sum_{j=1}^{k} \beta_{i_{j}}$.

An immediate corollary of this result is

Corollary 2.1 (Marginal pdf). Consider the pdf in equation (2.5). Then its marginal on $Q_{i}$ is given by

$$
\begin{aligned}
f_{Q_{i}}\left(q_{i}\right)= & q_{i}^{\nu \beta_{i}-1}\left(1-q_{i}\right)^{\nu\left(\bar{\beta}-\beta_{i}\right)-1} \\
& \times \int_{0}^{\infty} y^{\nu \bar{\beta}-1} E_{\nu, \nu \beta_{i}}^{\beta_{i}}\left(-\left(y q_{i}\right)^{\nu}\right) E_{\nu, \nu\left(\bar{\beta}-\beta_{i}\right)}^{\bar{\beta}-\beta_{i}}\left(\left(-y\left(1-q_{i}\right)^{\nu}\right)\right) \mathrm{d} y
\end{aligned}
$$

As the three-parameter Mittag-Leffler function has a representation as an $H$-function [15],

$$
H_{p, r}^{m, n}(z)=H_{p, r}^{m, n}\left[z \mid \begin{array}{l}
\left(a_{i}, A_{i}\right)_{1, p} \\
\left(b_{i}, B_{i}\right)_{1, r}
\end{array}\right],
$$

for suitable choices of $\left(a_{i}, A_{i}\right)$ and $\left(b_{i}, B_{i}\right)$, the marginal pdf (2.14) can be expressed in terms of an $H$-function too. 
A FRACTIONAL GENERALIZATION OF THE DIRICHLET... 119

Proposition 2.3. If $Q_{i}$ is the random variable with $p d f$ (2.14), then

$$
\begin{aligned}
& f_{Q_{i}}\left(q_{i}\right)=\frac{1}{\nu \Gamma\left(\beta_{i}\right) \Gamma\left(\bar{\beta}-\beta_{i}\right)} q_{i}^{\nu \beta_{i}-1}\left(1-q_{i}\right)^{-\left(\nu \beta_{i}+1\right)} \\
& \times \lim _{\varepsilon \downarrow 0} H_{3,3}^{2,2}\left[\left(\frac{q_{i}}{1-q_{i}}\right)^{\nu} \mid \begin{array}{ccc}
\left(1-\beta_{i}, 1\right) & (1-\bar{\beta}+\varepsilon, 1) & \left(\nu\left(\varepsilon-\beta_{i}\right), \nu\right) \\
(0,1) & \left(\varepsilon-\beta_{i}, 1\right) & \left(1-\nu \beta_{i}, \nu\right)
\end{array}\right] .
\end{aligned}
$$

P r o o f. In the integral (2.14) set

$$
\delta_{1}=\beta_{i}, \delta_{2}=\bar{\beta}-\beta_{i}, \bar{q}_{1}=q_{i}^{\nu}, \text { and } \bar{q}_{2}=\left(1-q_{i}\right)^{\nu} .
$$

Denote with $I_{\delta_{1}, \delta_{2}}^{\nu \bar{\beta}}$ the resulting integral and observe that

$$
I_{\delta_{1}, \delta_{2}}^{\nu \bar{\beta}}=\frac{1}{\nu} \int_{0}^{\infty} y^{\delta_{1}+\delta_{2}-1} E_{\nu, \nu \delta_{1}}^{\delta_{1}}\left(-y \bar{q}_{1}\right) E_{\nu, \nu \delta_{2}}^{\delta_{2}}\left(-y \bar{q}_{2}\right) \mathrm{d} y .
$$

For $\delta>0$ and $\nu \in(0,1]$ we have

$$
E_{\nu, \nu \delta}^{\delta}(z)=\frac{1}{\Gamma(\delta)} H_{1,2}^{1,1}\left[-z \mid \begin{array}{l}
(1-\delta, 1) \\
(0,1)(1-\nu \delta, \nu)
\end{array}\right] .
$$

For $\eta \in(0, \bar{\beta})$, by using (2.19) and Theorem 2.9 in [12], we have

$$
\begin{aligned}
I_{\delta_{1}, \delta_{2}}^{\eta} & =\int_{0}^{\infty} y^{\eta-1} E_{\nu, \nu \delta_{1}}^{\delta_{1}}\left(-y \bar{q}_{1}\right) E_{\nu, \nu \delta_{2}}^{\delta_{2}}\left(-y \bar{q}_{2}\right) \mathrm{d} y \\
& =\frac{\bar{q}_{2}^{-\eta}}{\Gamma\left(\delta_{1}\right) \Gamma\left(\delta_{2}\right)} H_{3,3}^{2,2}\left[\frac{\bar{q}_{1}}{\bar{q}_{2}} \mid \begin{array}{ccc}
\left(1-\delta_{1}, 1\right) & (1-\eta, 1) & \left(\nu\left(\delta_{2}-\eta\right), \nu\right) \\
(0,1) & \left(\delta_{2}-\eta, 1\right) & \left(1-\nu \delta_{1}, \nu\right)
\end{array}\right] .
\end{aligned}
$$

Set $\eta=\bar{\beta}-\varepsilon$ in (2.20) with $\varepsilon \in(0, \bar{\beta})$, and use (2.17) to recover $I_{\beta_{i}, \bar{\beta}-\beta_{i}}^{\bar{\beta}-\varepsilon}$. If $\varepsilon$ is sufficiently small, the poles $-l, \beta_{i}-\varepsilon-l,\left(\nu \beta_{i}-1-l\right) / \nu, l=0,1,2, \ldots$, do not coincide with the poles $\beta_{i}+k, \bar{\beta}-\varepsilon+k,\left(\nu\left(\varepsilon-\beta_{i}\right)+k\right) / \nu, k=0,1,2, \ldots$. Then, according to Theorem 1.1 in [12, the $H$-function in (2.16) makes sense for all $q_{i} \in(0,1)$ as $A_{1}+A_{2}-A_{3}+B_{1}+B_{2}-B_{3}=4-2 \nu>0$. The claim follows by taking the limit as $\varepsilon \downarrow 0$ of $I_{\beta_{i}, \bar{\beta}-\beta_{i}}^{\bar{\beta}}$.

Remark 2.4. By using Properties 2.1, 2.3 and 2.5 of [12, the $H$ function in (2.16) can be rewritten interchanging $\beta_{i}$ with $\bar{\beta}-\beta_{i}$ and $q_{i}$ with $1-q_{i}$, which corresponds to commuting the two Mittag-Leffler functions in (2.14). 
According to Theorems 1.3 and 1.4 in [12], since

$$
\begin{aligned}
& \sum_{i=1}^{3}\left(B_{i}-A_{i}\right)=0, \quad \prod_{i=1}^{3} \frac{B_{i}^{B_{i}}}{A_{i}^{A_{i}}}=1, \\
& \sum_{i=1}^{3}\left(b_{i}-a_{i}\right)=\bar{\beta}(1-\nu)+\nu(\bar{\beta}-\varepsilon)>0,
\end{aligned}
$$

the $H$-function in (2.16) has a power series expansion. The following propositions rely on this property.

Proposition 2.4. For $q_{i}<1 / 2$ and $\beta_{i}$ not a positive integer

$$
\begin{aligned}
& f_{Q_{i}}\left(q_{i}\right)=\frac{q_{i}^{\nu \beta_{i}-1}\left(1-q_{i}\right)^{-\left(\nu \beta_{i}+1\right)}}{\nu \Gamma\left(\beta_{i}\right) \Gamma\left(\bar{\beta}-\beta_{i}\right)} \\
& \times\left[\Gamma(\bar{\beta}) \frac{\Gamma\left(-\beta_{i}\right) \Gamma\left(\beta_{i}\right)}{\Gamma\left(-\nu \beta_{i}\right) \Gamma\left(\nu \beta_{i}\right)}+\sum_{k=1}^{\infty}(-1)^{k} D_{k}\left(\frac{q_{i}}{1-q_{i}}\right)^{\nu k}\right],
\end{aligned}
$$

where

$$
D_{k}=\frac{\Gamma(\bar{\beta}+k) \Gamma\left(-\beta_{i}-k\right) \Gamma\left(\beta_{i}+k\right)}{k ! \Gamma\left(-\nu\left(\beta_{i}+k\right)\right) \Gamma\left(\nu\left(\beta_{i}+k\right)\right)}+\frac{\left(1-q_{i}\right)^{\nu \beta_{i}} \Gamma\left(\beta_{i}-k\right) \Gamma\left(\bar{\beta}-\beta_{i}+k\right)}{q_{i}^{\nu \beta_{i}} \Gamma(-\nu k) \Gamma(\nu k)} .
$$

P r o o f. Consider the $H$-function $H_{3,3}^{2,2}$ in (2.16). If $\beta_{i}$ is not a positive integer, we have $B_{1}\left(b_{2}+l\right) \neq B_{2}\left(b_{1}+k\right)$ for $l, k=0,1,2, \ldots$ Thus, thanks to (2.21), from Theorem 1.3 of [12], $H_{3,3}^{2,2}$ is an analytical function in $q_{i}^{\nu} /\left(1-q_{i}\right)^{\nu}$ and has the following power series expansion for $q_{i}<1 / 2$ :

$$
\begin{aligned}
& \sum_{k=0}^{\infty} \frac{\Gamma\left(b_{2}-k\right) \Gamma\left(1-a_{1}+k\right) \Gamma\left(1-a_{2}+k\right)}{\Gamma\left(1-b_{3}+\nu k\right) \Gamma\left(a_{3}-\nu k\right)} \frac{(-1)^{k}}{k !}\left(\frac{q_{i}}{1-q_{i}}\right)^{\nu k}+\left(\frac{q_{i}}{1-q_{i}}\right)^{\nu b_{2}} \\
& +\sum_{k=0}^{\infty} \frac{\Gamma\left(-b_{2}-k\right) \Gamma\left(1-a_{1}+b_{2}+k\right) \Gamma\left(1-a_{2}+b_{2}+k\right)}{\Gamma\left(1-b_{3}+\nu\left(b_{2}+k\right)\right) \Gamma\left(a_{3}-\nu\left(b_{2}+k\right)\right)} \frac{(-1)^{k}}{k !}\left(\frac{q_{i}}{1-q_{i}}\right)^{\nu k} .
\end{aligned}
$$

The claim follows replacing $a_{1}=1-\beta_{i}, a_{2}=1-\bar{\beta}+\varepsilon, a_{3}=\nu\left(\varepsilon-\beta_{i}\right), b_{2}=$ $\varepsilon-\beta_{i}$ and $b_{3}=1-\nu \beta_{i}$ in (2.24) and taking the limit as $\varepsilon \downarrow 0$.

REMARK 2.5. If $\nu\left(\beta_{i}+k\right)$ is not a positive integer for $k=0,1,2, \ldots$ thanks to the reflection formula for the gamma function [12, we might simplify the expansion in (2.22) using 
A FRACTIONAL GENERALIZATION OF THE DIRICHLET... 121

$$
\frac{\Gamma\left(-\beta_{i}-k\right) \Gamma\left(\beta_{i}+k\right)}{\Gamma\left(-\nu\left(\beta_{i}+k\right)\right) \Gamma\left(\nu\left(\beta_{i}+k\right)\right)}=\nu \frac{\sin \left(\pi \nu\left(\beta_{i}+k\right)\right)}{\sin \left(\pi\left(\beta_{i}+k\right)\right)} .
$$

Similarly we get $\Gamma(-\nu k) \Gamma(\nu k)=-\pi /(k \nu \sin (\pi \nu k))$ if $\nu k$ is not a positive integer.

Proposition 2.5. For $q_{i}>1 / 2$ and $\bar{\beta}-\beta_{i}$ not a positive integer

$$
\begin{aligned}
& f_{Q_{i}}\left(q_{i}\right)=\frac{q_{i}^{-\left(\nu\left(\bar{\beta}-\beta_{i}\right)+1\right)}\left(1-q_{i}\right)^{\nu\left(\bar{\beta}-\beta_{i}\right)-1}}{\nu \Gamma\left(\beta_{i}\right) \Gamma\left(\bar{\beta}-\beta_{i}\right)} \\
& \times\left[\Gamma(\bar{\beta}) \frac{\Gamma\left(-\left(\bar{\beta}-\beta_{i}\right)\right) \Gamma\left(\bar{\beta}-\beta_{i}\right)}{\Gamma\left(-\nu\left(\bar{\beta}-\beta_{i}\right)\right) \Gamma\left(\nu\left(\bar{\beta}-\beta_{i}\right)\right)}+\sum_{k=1}^{\infty}(-1)^{k} D_{k}\left(\frac{1-q_{i}}{q_{i}}\right)^{\nu k}\right],
\end{aligned}
$$

where

$$
\begin{aligned}
D_{k}= & \frac{\Gamma(\bar{\beta}+k)}{k !} \frac{\Gamma\left(-\left(\bar{\beta}-\beta_{i}+k\right)\right) \Gamma\left(\bar{\beta}-\beta_{i}+k\right)}{\Gamma\left(-\nu\left(\bar{\beta}-\beta_{i}+k\right)\right) \Gamma\left(\nu\left(\bar{\beta}-\beta_{i}+k\right)\right)} \\
& +\left(\frac{q_{i}}{1-q_{i}}\right)^{\nu\left(\bar{\beta}-\beta_{i}\right)} \frac{\Gamma\left(\beta_{i}+k\right) \Gamma\left(\bar{\beta}-\beta_{i}-k\right)}{\Gamma(-\nu k) \Gamma(\nu k)} .
\end{aligned}
$$

P r o o f. Consider again the $H$-function $H_{3,3}^{2,2}$ in (2.16). If $\bar{\beta}-\beta_{i}$ is not a positive integer, we have $A_{1}\left(1-a_{2}+l\right) \neq A_{2}\left(1-a_{1}+k\right)$ for $l, k=0,1,2, \ldots$ From (2.21) and Theorem 1.4 of [12], $H_{3,3}^{2,2}$ is an analytical function in $q_{i}^{\nu} /\left(1-q_{i}\right)^{\nu}$ and has the following power series expansion for $q_{i}>1 / 2$ :

$\sum_{k=0}^{\infty} \frac{\Gamma\left(1-a_{1}+k\right) \Gamma\left(b_{2}+1-a_{1}+k\right) \Gamma\left(a_{1}-a_{2}-k\right)}{\Gamma\left(a_{3}+\nu\left(1-a_{1}+k\right)\right) \Gamma\left(1-b_{3}-\nu\left(1-a_{1}+k\right)\right)} \frac{(-1)^{k}}{k !}\left[\frac{q_{i}}{1-q_{i}}\right]^{\nu\left(a_{1}-1-k\right)}$
$+\sum_{k=0}^{\infty} \frac{\Gamma\left(1-a_{2}+k\right) \Gamma\left(b_{2}+1-a_{2}+k\right) \Gamma\left(a_{2}-a_{1}-k\right)}{\Gamma\left(a_{3}+\nu\left(1-a_{2}+k\right)\right) \Gamma\left(1-b_{3}-\nu\left(1-a_{2}+k\right)\right)} \frac{(-1)^{k}}{k !}\left[\frac{q_{i}}{1-q_{i}}\right]^{\nu\left(a_{2}-1-k\right)}$

The claim follows replacing $a_{1}=1-\beta_{i}, a_{2}=1-\bar{\beta}+\varepsilon, a_{3}=\nu\left(\varepsilon-\beta_{i}\right), b_{2}=$ $\varepsilon-\beta_{i}$ and $b_{3}=1-\nu \beta_{i}$ in (2.28) and taking the limit as $\varepsilon \downarrow 0$.

By using the reflection formula for the gamma function, also the expansion (2.26) might be simplified similarly to what has been addressed in Remark 2.5. 


\section{An alternative generalization}

We now give an alternative generalization with desirable properties which in addition approximates the fractional Dirichlet distribution with density (2.5) for appropriate values of the parameters.

Let us thus consider a random vector $\boldsymbol{Q}=\left(Q_{1}, \ldots, Q_{n-1}\right), n \geq 2$, with the following probability density function:

$$
\begin{aligned}
f_{\boldsymbol{Q}}\left(q_{1}, \ldots, q_{n-1}\right)= & \left(\prod_{i=1}^{n-1} q_{i}^{\nu \beta_{i}-1}\right)\left(1-\sum_{i=1}^{n-1} q_{i}\right)^{\nu \beta_{n}-1} \\
& \times \frac{\nu^{n-1} \Gamma(\bar{\beta})}{\Gamma\left(\beta_{1}\right) \cdot \ldots \cdot \Gamma\left(\beta_{n}\right)}\left(q_{1}^{\nu}+\ldots+\left(1-\sum_{i=1}^{n-1} q_{i}\right)^{\nu}\right)^{-\bar{\beta}}
\end{aligned}
$$

for $q_{1}, \ldots, q_{n-1} \in(0,1), q_{1}+\ldots+q_{n-1}<1, \nu>0, \beta_{i}>0, i=1, \ldots, n$, $\bar{\beta}=\beta_{1}+\cdots+\beta_{n}$.

For the sake of clarity we check that $f_{\boldsymbol{Q}}\left(q_{1}, \ldots, q_{n-1}\right)$ as given in (3.1) is a genuine probability density function. This will follow by proving that

$$
\frac{\nu^{n-1} \Gamma(\bar{\beta})}{\Gamma\left(\beta_{1}\right) \cdot \ldots \cdot \Gamma\left(\beta_{n}\right)}
$$

in the rhs of (3.1) plays the role of a normalization coefficient.

THEOREM 3.1. We have

$$
\begin{aligned}
\int_{0}^{1} \mathrm{~d} q_{1} \cdots & \int_{0}^{1-q_{1}-\cdots-q_{n-2}} \mathrm{~d} q_{n-1}\left(\prod_{i=1}^{n-1} q_{i}^{\nu \beta_{i}-1}\right)\left(1-\sum_{i=1}^{n-1} q_{i}\right)^{\nu \beta_{n}-1} \\
& \times\left(q_{1}^{\nu}+\ldots+\left(1-\sum_{i=1}^{n-1} q_{i}\right)^{\nu}\right)^{-\bar{\beta}}=\frac{\Gamma\left(\beta_{1}\right) \cdot \ldots \cdot \Gamma\left(\beta_{n}\right)}{\nu^{n-1} \Gamma(\bar{\beta})} .
\end{aligned}
$$

P r o o f. Observe that the lhs of (3.3) can be rewritten as

$$
\begin{array}{rl}
I=\int_{0}^{1} & \mathrm{~d} q_{1} \cdots \int_{0}^{1-q_{1}-\cdots-q_{n-2}} \mathrm{~d} q_{n-1}\left(\prod_{i=1}^{n-1} q_{i}^{-1}\right) \\
& \times(1-\bar{q})^{-1} \prod_{i=1}^{n-1}\left(\frac{q_{i}}{1-\bar{q}}\right)^{\nu \beta_{i}}\left(1+\sum_{i=1}^{n-1}\left(\frac{q_{i}}{1-\bar{q}}\right)^{\nu}\right)^{-\bar{\beta}},
\end{array}
$$


A FRACTIONAL GENERALIZATION OF THE DIRICHLET... 123

where $\bar{q}=q_{1}+\cdots+q_{n-1}$. Apply the change of variables $(1-\bar{q}) / q_{i}=z_{i}$ for $i=1, \ldots, n-1$, in multivariate integration. Thus, we have

$$
\begin{aligned}
& q_{i}=\frac{\prod_{j \in \mathcal{I}_{n-1, i}} z_{j}}{\prod_{j=1}^{n-1} z_{j}+\sum_{k=1}^{n-1} \prod_{j \in \mathcal{I}_{n-1, k}} z_{j}}, \quad i=1, \ldots, n-1, \\
& J=\frac{\prod_{j=1}^{n-1} z_{j}^{n-2}}{\left(\prod_{j=1}^{n-1} z_{j}+\sum_{k=1}^{n-1} \prod_{j \in \mathcal{I}_{n-1, k}} z_{j}\right)^{n}},
\end{aligned}
$$

where $\mathcal{I}_{n-1, k}=\{1, \ldots, k-1, k+1, \ldots, n-1\}$ for $k=1, \ldots, n-1$, and $J$ is the Jacobian of the transformation. Note that

$$
1-\bar{q}=\frac{\prod_{j=1}^{n-1} z_{j}}{\left(\prod_{j=1}^{n-1} z_{j}+\sum_{k=1}^{n-1} \prod_{j \in \mathcal{I}_{n-1, k}} z_{j}\right)} .
$$

By putting (3.5) and (3.6) in (3.4) we have

$$
I=\int_{0}^{\infty} \mathrm{d} z_{1} \cdots \int_{0}^{\infty} \mathrm{d} z_{n-1} \prod_{i=1}^{n-1} z_{j}^{-\nu \beta_{j}-1}\left(1+\sum_{j=1}^{n-1} \frac{1}{z_{j}^{\nu}}\right)^{-\bar{\beta}}
$$

Apply the change of variables $z_{i}^{\nu}=t_{i}$ for $i=1, \ldots, n-1$, in multivariate integration. Then, we have $z_{i}=t_{i}^{1 / \nu}$ for $i=1, \ldots, n-1$, and $\nu^{1-n} \prod_{i=1}^{n-1} t_{i}^{1 / \nu-1}$ is the Jacobian of this transformation. From (3.7)

$$
I=\frac{1}{\nu^{n-1}} \int_{0}^{\infty} \mathrm{d} t_{1} \cdots \int_{0}^{\infty} \mathrm{d} t_{n-2} I_{n-2}\left(t_{1}, \ldots, t_{n-2}\right),
$$

where

$$
I_{n-2}\left(t_{1}, \ldots, t_{n-2}\right)=\int_{0}^{\infty} \mathrm{d} t_{n-1} \prod_{i=1}^{n-1} t_{j}^{-\beta_{j}-1}\left(1+\sum_{j=1}^{n-1} \frac{1}{t_{j}}\right)^{-\bar{\beta}} .
$$

Observe that $I_{n-2}\left(t_{1}, \ldots, t_{n-2}\right)$ in (3.9) can be rewritten as

$$
\begin{aligned}
I_{n-2} & \left(t_{1}, \ldots, t_{n-2}\right) \\
= & \int_{0}^{\infty} \mathrm{d} t_{n-1} \prod_{j=1}^{n-1} t_{j}^{\bar{\beta}-\beta_{j}-1}\left(\prod_{j=1}^{n-1} t_{j}+\sum_{k=1}^{n-1} \prod_{j \in \mathcal{I}_{n-1, k}} t_{j}\right)^{-\bar{\beta}} \\
= & \prod_{i=1}^{n-2} t_{i}^{-\beta_{i}-1} \\
& \times \int_{0}^{\infty} \mathrm{d} t_{n-1}\left(1+t_{n-1} \frac{\prod_{i=1}^{n-2} t_{i}+\sum_{k=1}^{n-2} \prod_{i \in \mathcal{I}_{n-2, k}} t_{i}}{\prod_{i=1}^{n-2} t_{i}}\right)^{-\bar{\beta}} t_{n-1}^{\bar{\beta}-\beta_{n-1}-1} .
\end{aligned}
$$


With the change of variable $z=t_{n-1}\left(\prod_{i=1}^{n-2} t_{i}+\sum_{k=1}^{n-2} \prod_{i \in \mathcal{I}_{n-2, k}} t_{i}\right) / \prod_{i=1}^{n-2} t_{i}$ and by recalling the Mellin trasform of $(1+z)^{-\bar{\beta}}$, we recover

$$
\begin{aligned}
& I_{n-2}\left(t_{1}, \ldots, t_{n-2}\right) \\
& =\frac{\prod_{i=1}^{n-2} t_{i}^{\bar{\beta}-\beta_{i}-\beta_{n-1}-1}}{\left(\prod_{i=1}^{n-2} t_{i}+\sum_{k=1}^{n-2} \prod_{i \in \mathcal{I}_{n-2, k}} t_{i}\right)^{\bar{\beta}-\beta_{n-1}}} \frac{\Gamma\left(\bar{\beta}-\beta_{n-1}\right) \Gamma\left(\beta_{n-1}\right)}{\Gamma(\bar{\beta})} .
\end{aligned}
$$

Now, replace $I_{n-2}\left(t_{1}, \ldots, t_{n-2}\right)$ in (3.8) with the closed form (3.11). This leads us to

$$
I=\frac{1}{\nu^{n-1}} \frac{\Gamma\left(\bar{\beta}-\beta_{n-1}\right) \Gamma\left(\beta_{n-1}\right)}{\Gamma(\bar{\beta})} \int_{0}^{\infty} \mathrm{d} t_{1} \ldots \int_{0}^{\infty} \mathrm{d} t_{n-3} I_{n-3}\left(t_{1}, \ldots, t_{n-3}\right),
$$

where

$$
\begin{aligned}
& I_{n-3}\left(t_{1}, \ldots, t_{n-3}\right) \\
& =\int_{0}^{\infty} \mathrm{d} t_{n-2} \prod_{j=1}^{n-2} t_{j}^{\bar{\beta}-\beta_{n-1}-\beta_{j}-1}\left(\prod_{j=1}^{n-2} t_{j}+\sum_{k=1}^{n-2} \prod_{j \in \mathcal{I}_{n-2, k}} t_{j}\right)^{-\left(\bar{\beta}-\beta_{n-1}\right)} .
\end{aligned}
$$

By comparing the integral in (3.13) with that in (3.10), we observe that the former has the same expression of the latter with $\bar{\beta}$ replaced by $\bar{\beta}-$ $\beta_{n-1}$. Thus, by recurring to the same arguments employed to compute $I_{n-2}\left(t_{1}, \ldots, t_{n-2}\right)$ we recover

$$
\begin{aligned}
I_{n-3}\left(t_{1}, \ldots, t_{n-3}\right)= & \frac{\prod_{i=1}^{n-3} t_{i}^{\bar{\beta}-\beta_{n-1}-\beta_{n-2}-\beta_{i}-1}}{\left(\prod_{i=1}^{n-3} t_{i}+\sum_{k=1}^{n-3} \prod_{i \in \mathcal{I}_{n-3, k}} t_{i}\right)^{\bar{\beta}-\beta_{n-1}-\beta_{n-2}}} \\
& \times \frac{\Gamma\left(\bar{\beta}-\beta_{n-1}-\beta_{n-2}\right) \Gamma\left(\beta_{n-2}\right)}{\Gamma\left(\bar{\beta}-\beta_{n-1}\right)} .
\end{aligned}
$$

Replacing $I_{n-3}\left(t_{1}, \ldots, t_{n-3}\right)$ in (3.12) with the closed form (3.14) we get

$$
\begin{aligned}
I= & \frac{1}{\nu^{n-1}} \frac{\Gamma\left(\bar{\beta}-\beta_{n-1}-\beta_{n-2}\right) \Gamma\left(\beta_{n-1}\right) \Gamma\left(\beta_{n-2}\right)}{\Gamma(\bar{\beta})} \\
& \times \int_{0}^{\infty} \mathrm{d} t_{1} \cdots \int_{0}^{\infty} \mathrm{d} t_{n-4} I_{n-4}\left(t_{1}, \ldots, t_{n-4}\right),
\end{aligned}
$$

where

$$
I_{n-4}\left(t_{1}, \ldots, t_{n-4}\right)=\int_{0}^{\infty} \mathrm{d} t_{n-3} \prod_{j=1}^{n-3} t_{j}^{\bar{\beta}-\beta_{n-1}-\beta_{n-2}-\beta_{j}-1}
$$


A FRACTIONAL GENERALIZATION OF THE DIRICHLET... 125

$$
\times\left(\prod_{j=1}^{n-3} t_{j}+\sum_{k=1}^{n-3} \prod_{j \in \mathcal{I}_{n-3, k}} t_{j}\right)^{-\left(\bar{\beta}-\beta_{n-1}-\beta_{n-2}\right)}
$$

which indeed has the same expression of $I_{n-2}$ and $I_{n-3}$ with suitable updates of $\bar{\beta}$. The result follows by iterating from $i=4$ up to $i=n-1$ the computation of

$$
\begin{aligned}
I= & \frac{1}{\nu^{n-1}} \frac{\Gamma\left(\bar{\beta}-\sum_{k=n-i+2}^{n-1} \beta_{k}\right) \prod_{k=n-i+2}^{n-1} \Gamma\left(\beta_{k}\right)}{\Gamma(\bar{\beta})} \\
& \times \int_{0}^{\infty} \mathrm{d} t_{1} \cdots \int_{0}^{\infty} \mathrm{d} t_{n-i} I_{n-i}\left(t_{1}, \ldots, t_{n-i}\right)
\end{aligned}
$$

with

$$
\begin{aligned}
I_{n-i}\left(t_{1}, \ldots, t_{n-i}\right)= & \int_{0}^{\infty} \mathrm{d} t_{n-i+1} \prod_{j=1}^{n-i+1} t_{j}^{\bar{\beta}-\sum_{k=n-i+2}^{n-1} \beta_{k}-\beta_{j}-1} \\
& \times\left(\prod_{j=1}^{n-i+1} t_{j}+\sum_{k=1}^{n-i+1} \prod_{j \in \mathcal{I}_{n-i+1, k}} t_{j}\right)^{-\left(\bar{\beta}-\sum_{k=n-i+2}^{n-1} \beta_{k}\right)}
\end{aligned}
$$

We obtain the closed form expression

$$
\begin{aligned}
I_{n-i}\left(t_{1}, \ldots, t_{n-i}\right)= & \frac{\prod_{j=1}^{n-i} t_{j}^{\bar{\beta}-\sum_{k=n-i+1}^{n-1} \beta_{k}-\beta_{j}-1}}{\left(\prod_{i=1}^{n-i} t_{i}+\sum_{k=1}^{n-i} \prod_{i \in \mathcal{I}_{n-i, k}} t_{i}\right)^{\bar{\beta}-\sum_{k=n-i+1}^{n-1} \beta_{k}}} \\
& \times \frac{\Gamma\left(\bar{\beta}-\sum_{k=n-i+i}^{n-1} \beta_{k}\right) \Gamma\left(\beta_{n-i+1}\right)}{\Gamma\left(\bar{\beta}-\sum_{k=n-i+2}^{n-1} \beta_{k}\right)}
\end{aligned}
$$

with $\sum_{k=1}^{n-i} \prod_{i \in \mathcal{I}_{n-i, k}} t_{i}=1$ for $i=n-1$. The last replacement with $I_{1}\left(t_{1}\right)$ gives

$$
I=\frac{1}{\nu^{n-1}} \frac{\Gamma\left(\beta_{1}+\beta_{n}\right) \Gamma\left(\beta_{2}\right) \cdot \ldots \cdot \Gamma\left(\beta_{n-1}\right)}{\Gamma(\bar{\beta})} \int_{0}^{\infty} \mathrm{d} t_{1}\left(1+t_{1}\right)^{-\left(\beta_{1}+\beta_{n}\right)} t_{1}^{\beta_{n}-1}
$$

from which the claimed result follows by observing that

$$
\int_{0}^{\infty} \mathrm{d} t_{1} t_{1}^{\beta_{n}-1}\left(1+t_{1}\right)^{-\left(\beta_{1}+\beta_{n}\right)}=\Gamma\left(\beta_{n}\right) \Gamma\left(\beta_{1}\right) / \Gamma\left(\beta_{1}+\beta_{n}\right) .
$$


REMARK 3.1. Alternatively, in (3.7) use the transformation $z_{1}^{-1}=$ $t_{1}, \ldots, z_{n}^{-1}=t_{n}$. Then, we have (cf. [9] no. 4.638/3, p. 649)

$$
\begin{aligned}
I & =\int_{0}^{\infty} \mathrm{d} t_{1} \cdots \int_{0}^{\infty} \mathrm{d} t_{n-1} \frac{\prod_{i=1}^{n-1} t_{j}^{\nu \beta_{j}-1}}{\left(1+t_{1}^{\nu}+\cdots+t_{n-1}^{\nu}\right)^{\bar{\beta}}} \\
& =\frac{\Gamma\left(\beta_{1}\right) \cdot \ldots \cdot \Gamma\left(\beta_{n-1}\right)}{\nu^{n-1}} \frac{\Gamma\left(\bar{\beta}-\beta_{1}-\cdots-\beta_{n-1}\right)}{\Gamma(\bar{\beta})},
\end{aligned}
$$

which is in agreement with (3.3).

REMARK 3.2. On the $n$-dimensional simplex $\Delta_{n}$ the pdf of the random vector $\left(Q_{1}, \ldots, Q_{n}\right)$, where $\sum_{n} Q_{n}=1$ a.s., writes

$$
\begin{aligned}
& \mathbb{P}\left(\left(Q_{1}, \ldots, Q_{n}\right) \in \mathrm{d}\left(q_{1}, \ldots, q_{n}\right)\right) \\
& =\frac{\nu^{n-1} \Gamma(\bar{\beta})}{\Gamma\left(\beta_{1}\right) \cdot \ldots \cdot \Gamma\left(\beta_{n}\right)}\left(q_{1}^{\nu}+\ldots+q_{n}^{\nu}\right)^{-\bar{\beta}} \prod_{i=1}^{n} q_{i}^{\nu \beta_{i}-1} \\
& =\frac{\nu^{n-1}}{B(\boldsymbol{\beta})} \prod_{i=1}^{n} q_{i}^{-1}\left(\frac{q_{i}^{\nu}}{\sum_{i=1}^{n} q_{i}^{\nu}}\right)^{\beta_{i}},
\end{aligned}
$$

with $B(\boldsymbol{\beta})=\prod_{i=1}^{n} \Gamma\left(\beta_{i}\right) / \Gamma\left(\sum_{i=1}^{n} \beta_{i}\right)$. In short we write $\left(Q_{1}, \ldots, Q_{n}\right) \sim$ $\operatorname{GDIR}(\nu, \boldsymbol{\beta})$.

Notice that for $\nu=1$ the $\operatorname{Dirichlet}(\boldsymbol{\beta})$ is obtained. In this case the random vector $\left(Q_{1}, \ldots, Q_{n}\right)$ is uniformly distributed on $\Delta_{n}$ for $\beta_{i}=1$, $i \in \mathbb{N}^{*}$. If instead we only let $\beta_{i}=1$,

$$
\mathbb{P}\left(\left(Q_{1}, \ldots, Q_{n}\right) \in \mathrm{d}\left(q_{1}, \ldots, q_{n}\right)\right)=\frac{\nu^{n-1}(n-1) !}{\left(q_{1}^{\nu}+\ldots+q_{n}^{\nu}\right)^{n}} \prod_{i=1}^{n} q_{i}^{\nu-1}
$$

which is symmetric but clearly not uniform. If $\beta_{i}=1 / \nu$ (again symmetric) we obtain

$$
\mathbb{P}\left(\left(Q_{1}, \ldots, Q_{n}\right) \in \mathrm{d}\left(q_{1}, \ldots, q_{n}\right)\right)=\frac{\nu^{n-1} \Gamma(n / \nu)}{\Gamma(1 / \nu)^{n}}\left(q_{1}^{\nu}+\cdots+q_{n}^{\nu}\right)^{-n / \nu} .
$$

REMARK 3.3. The alternative generalized Dirichlet distribution considered in this section (i.e. that with pdf (3.1)) can be derived by the same procedure described in Section 2 with $\left(Z_{i}\right)^{\nu}$ distributed as $\operatorname{Gamma}\left(\beta_{i}, 1\right)$, $i=1, \ldots, n-1$. Note that the random variable $X$ such that $X^{\nu}, \nu>0$, is $\operatorname{Gamma}(\alpha, 1)$-distributed, $\alpha>0$, is a special case of the generalized Gamma distribution (see e.g. [11, Section 8.7). In particular, $X$ has pdf 
A FRACTIONAL GENERALIZATION OF THE DIRICHLET... 127

$$
f_{X}(x)=\nu \frac{x^{\nu \alpha-1} e^{-x^{\nu}}}{\Gamma(\alpha)} \mathbf{1}_{\mathbb{R}_{+}},
$$

and Laplace transform (from (2.3.23) of [15] and the definition of Wright functions)

$$
\mathbb{E} e^{-z X}=\frac{\nu z^{-\nu \alpha}}{\Gamma(\alpha)} \sum_{k=0}^{\infty} \frac{\left(-z^{-\nu}\right)^{k}}{k !} \Gamma(\nu(k+\alpha)) .
$$

REMARK 3.4. The generalized Dirichlet pdf (3.1) turns out to be a reasonably good approximation of the fractional Dirichlet pdf (2.5) for $\beta_{i}<$ 1 (see for example Fig. 3). A partial explanation is that for $\lambda=1, \beta<1$, and $\nu \in(0,1]$ the fractional Gamma pdf (2.1) has a rather similar shape to the generalized Gamma pdf (3.25), as Fig. 1 shows for $\beta=0.2$ and $\beta=0.4$. For $\beta_{i}>1$, the fractional Dirichlet pdf exhibits a behaviour different from the generalized Dirichlet pdf (see for example Fig. 2). Indeed, Fig. 1 shows a different shape of the fractional Gamma pdf compared to the generalized Gamma pdf for $\beta=2$ and $\beta=3$.

Proposition 3.1 (Conjugate distribution). The generalized Dirichlet distribution $\operatorname{GDIR}(\nu, \boldsymbol{\beta})$ (with pdf (3.22) ) is the conjugate prior to a reparametrized Multinomial distribution with pmf

$$
\frac{N}{x_{1} ! \cdot \ldots \cdot x_{n} !}\left(\prod_{i=1}^{n} q_{i}^{\nu x_{i}}\right)\left(q_{1}^{\nu}+\ldots+q_{n}^{\nu}\right)^{-\sum_{i=1}^{n} x_{i}}
$$

where $N \in \mathbb{N}^{+}, x_{i} \in\{0, \ldots, N\}, i=1, \ldots, n, n \in \mathbb{N}^{+}, q_{1}+\ldots+q_{n}=1$, $\nu>0$. In particular, if the prior is $\operatorname{GDIR}(\nu, \boldsymbol{\beta})$ and the likelihood is as in (3.27), then the posterior becomes $\operatorname{GDIR}(\nu, \boldsymbol{\beta}+\boldsymbol{x})$.

P r o o f. The proof is a straightforward application of Bayes theorem. The reparametrization in (3.27) is such that $p_{i}=q_{i}^{\nu} /\left(\sum_{i=1}^{n} q_{i}^{\nu}\right)$, $i=1, \ldots, n$, are the event probabilities (i.e. $\sum_{i=1}^{n} p_{i}=1$ ).

3.1. Representation in terms of Dirichlet random variables. In order to derive a meaningful representation in terms of Dirichlet random variables for the random vector $\boldsymbol{Q}$, we first recall the definitions of two related classes of random vectors (see [10]).

Definition 3.1 (Liouville distribution of the first kind). Let $\boldsymbol{X}=$ $\left(X_{1}, \ldots, X_{n}\right)$ be an absolutely continuous random vector supported on the 

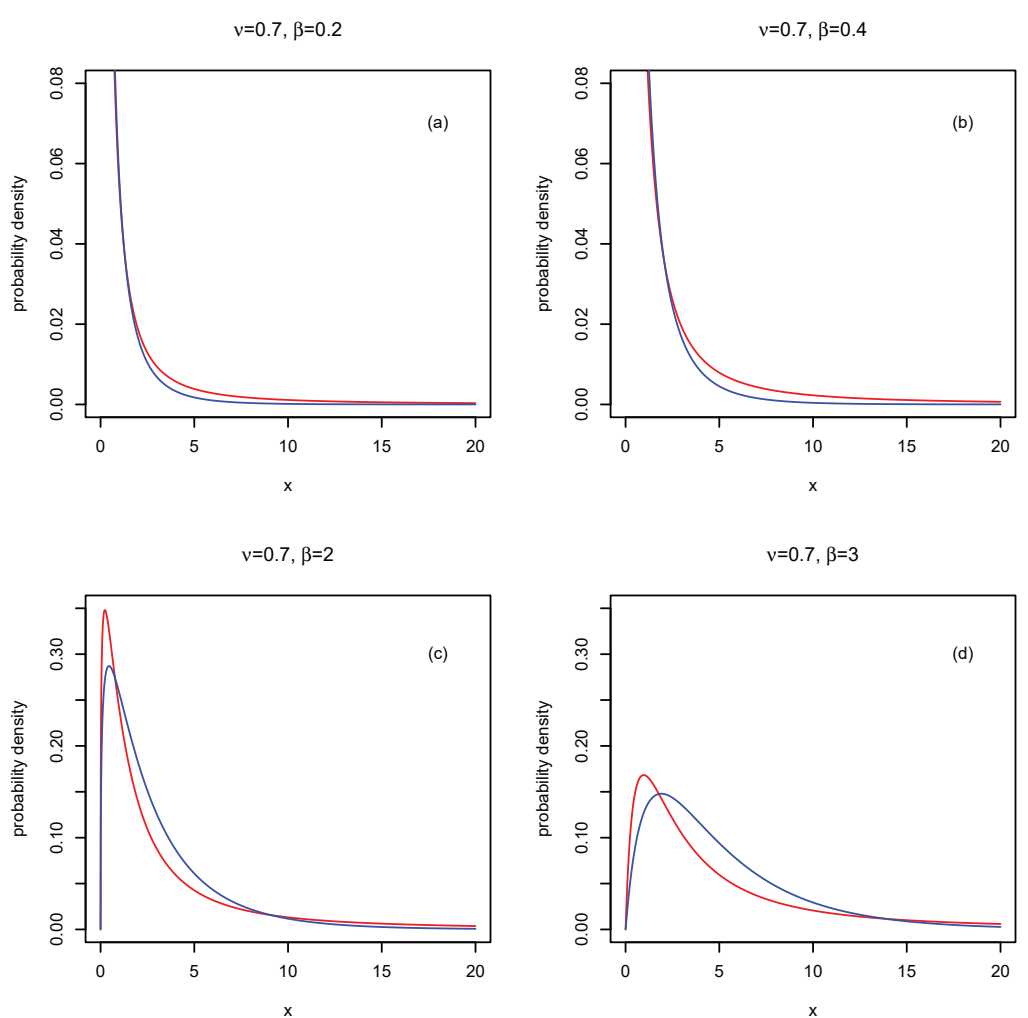

Figure 1. Comparison of the fractional Gamma pdf (2.1) (red line) versus the generalized Gamma pdf (3.25) (blue line) for $\nu=0.7$ and $\beta=0.2$ in (a) and $\beta=0.4$ in (b), as in Fig. 3, $\beta=2$ in (c) and $\beta=3$ in (d), as in Fig. 2,

$n$-dimensional positive orthant, i.e. $R^{n}=\left\{\left(x_{1}, \ldots, x_{n}\right): x_{i}>0\right.$ for each $i=$ $1, \ldots, n\}$. It is said to have Liouville distribution of the first kind if its joint pdf writes

$$
f_{\boldsymbol{X}}\left(x_{1}, \ldots, x_{n}\right) \propto f\left(\sum_{i=1}^{n} x_{i}\right) \prod_{i=1}^{n} x_{i}^{a_{i}-1}
$$

where $a_{i}>0, i=1, \ldots, n$, and $f$ is a positive continuous function satisfying $\int_{\mathbb{R}_{+}} y^{a-1} f(y) \mathrm{d} y<\infty$, with $a=a_{1}, \ldots, a_{n}$. Further, we write $\boldsymbol{X} \sim L_{n}^{(1)}\left[f(\cdot) ; a_{1}, \ldots, a_{n}\right]$. 
A FRACTIONAL GENERALIZATION OF THE DIRICHLET... 129

Definition 3.2 (Liouville distribution of the second kind). Let $\boldsymbol{Z}=$ $\left(Z_{1}, \ldots, Z_{n}\right)$ be an absolutely continuous random vector supported on $S_{n}=$ $\left\{\left(z_{1}, \ldots, z_{n}\right): z_{i}>0\right.$ for each $\left.i=1, \ldots, n, \sum_{i=1}^{n} z_{i}<1\right\}$. It is said to have Liouville distribution of the second kind if its joint pdf writes

$$
f_{\boldsymbol{Z}}\left(z_{1}, \ldots, z_{n}\right) \propto g\left(\sum_{i=1}^{n} z_{i}\right) \prod_{i=1}^{n} z_{i}^{c_{i}-1},
$$

where $c_{i}>0, i=1, \ldots, n$, and $g$ is a positive continuous function satisfying $\int_{\mathbb{R}_{+}} y^{c-1} g(y) \mathrm{d} y<\infty$, with $c=c_{1}, \ldots, c_{n}$. Further, we write $\boldsymbol{Z} \sim$ $L_{n}^{(2)}\left[g(\cdot) ; c_{1}, \ldots, c_{n}\right]$.

REMARK 3.5. If we let $f(t)=(1+t)^{-\left(a+a_{n+1}\right)}, t>0, a_{n+1}>0$, in (3.28), then $\boldsymbol{X}$ is distributed as an inverted Dirichlet.

If, in (3.29), we choose $g(t)=(1-t)^{c_{n+1}-1}, 0<t<1, a_{n+1}>0$, we have that $\boldsymbol{Z}$ is distributed as a Dirichlet.

Proposition 3.1 of [10] tells us what is the relationship between Liouville distributions of the first and of the second kind (and hence between the Dirichlet and the inverted Dirichlet). Specifically, if $\boldsymbol{Z} \sim L_{n}^{(2)}\left[g(\cdot) ; c_{1}, \ldots, c_{n}\right]$ and we consider the transformation

$$
X_{i}=\frac{Z_{i}}{1-\sum_{i=1}^{n} Z_{i}}, \quad i=1, \ldots, n
$$

then $\boldsymbol{X} \sim L_{n}^{(1)}\left[f(\cdot) ; c_{1}, \ldots, c_{n}\right]$, where

$$
f(t)=(1+t)^{-(c+1)} g\left(\frac{t}{1+t}\right), \quad t>0 .
$$

Plainly, the converse relation is true as well: inverting (3.31) (letting $h=$ $t /(1+t))$ we have

$$
g(h)=\left(\frac{1}{1-h}\right) f\left(\frac{h}{1-h}\right), \quad 0<h<1 .
$$

As a simple example, considering $f(t)=(1+t)^{-\left(c+c_{n+1}\right)}, t>0$ (inverted Dirichlet), we readily obtain $g(1-h)^{c_{n+1}-1}$ (Dirichlet).

Now, by exploiting the above definition we prove the following distributional representation for $\boldsymbol{Q}$. 
Proposition 3.2. Let $\boldsymbol{Q}=\left(Q_{1}, \ldots, Q_{n-1}\right)$ be distributed with $p d f$ (3.1). Then the random vector $\boldsymbol{M}=\left(M_{1}, \ldots, M_{n-1}\right)$ such that

$$
M_{i}=\frac{\left(\frac{Q_{i}}{1-\sum_{i=1}^{n-1} Q_{i}}\right)^{\nu}}{1+\left(\frac{Q_{i}}{1-\sum_{i=1}^{n-1} Q_{i}}\right)^{\nu}}, \quad i=1, \ldots, n-1,
$$

is distributed as a $\operatorname{Dirichlet}\left(\boldsymbol{\beta}=\left(\beta_{1}, \ldots, \beta_{n}\right)\right)$. that

Conversely, if $\boldsymbol{M} \sim \operatorname{Dirichlet}(\boldsymbol{\beta})$ we have that $\boldsymbol{Q}=\left(Q_{1}, \ldots, Q_{n-1}\right)$ such

$$
Q_{i}=\frac{\left(\frac{M_{i}}{1-\sum_{i=1}^{n-1} M_{i}}\right)^{\frac{1}{\nu}}}{1+\left(\frac{M_{i}}{1-\sum_{i=1}^{n-1} M_{i}}\right)^{\frac{1}{\nu}}}, \quad i=1, \ldots, n-1,
$$

is distributed with pdf (3.1). that

P r o o f. Let us define the random vector $\boldsymbol{Y}=\left(Y_{1}, \ldots, Y_{n-1}\right)$ such

$$
Y_{i}=\left(\frac{Q_{i}}{1-\sum_{i=1}^{n-1} Q_{i}}\right)^{\nu}, \quad i=1, \ldots, n-1,
$$

and let $\beta^{*}=\beta_{1}+\ldots+\beta_{n-1}$. Combining the transformations in the proof of Theorem 3.3 and of Remark 3.1 we see that $\boldsymbol{Y}$ has pdf

$$
f_{\boldsymbol{Y}}\left(y_{1}, \ldots, y\right)=\frac{\Gamma\left(\beta^{*}+\beta_{n}\right)}{\Gamma\left(\beta_{1}\right) \cdot \ldots \cdot \Gamma\left(\beta_{n}\right)}\left(1+\sum_{i=1}^{n-1} y_{i}\right)^{-\beta^{*}-\beta_{n}} \prod_{i=1}^{n-1} y_{i}^{\beta_{i}-1}
$$

and hence $\boldsymbol{Y} \sim L_{n-1}^{(1)}\left[(1+\cdot)^{-\left(\beta^{*}+\beta_{n}\right)} ; \beta_{1}, \ldots, \beta_{n-1}\right]$ (i.e. an inverted Dirichlet distribution).

By using the mentioned transformations between Liouville distributions of first and second kinds we have that $\boldsymbol{M} \sim L_{n-1}^{(2)}\left[(1-\cdot)^{\beta_{n-1}} ; \beta_{1}, \ldots, \beta_{n-1}\right]$ (Dirichlet), where

$$
M_{i}=\frac{Y_{i}}{1+\sum_{i=1}^{n-1} Y_{i}}, \quad i=1, \ldots, n-1,
$$

and (3.33) follows.

Finally, a rewriting of the components of $\boldsymbol{Q}$ in terms of those of $\boldsymbol{Y}$, leads easily to (3.34). 


\section{A FRACTIONAL GENERALIZATION OF THE DIRICHLET... 131}

\section{Monte Carlo simulations}

The simulation of the random variables $\boldsymbol{Q}$ for the fractional Dirichlet distribution is straightforward based on the construction presented in Section 2. First, one needs to generate random variables with density (2.1) and one can use the mixture representation discussed in [4]

$$
X_{i} \stackrel{d}{=} U_{i}^{1 / \nu} V_{\nu},
$$

where $U_{i}$ is $\operatorname{Gamma}\left(\beta_{i}, \lambda\right)$-distributed and $V_{\nu}$ is strictly positive-stable distributed with $\exp \left(-s^{\nu}\right)$ as the Laplace transform of the pdf. Summing the $X_{i}$ to get $W$ and dividing $X_{i}$ by $W$ gives $Q_{i}$.

REMARK 4.1. For $\beta=1$, there is an alternative representation [13, 6]:

$$
X \stackrel{d}{=} \Xi Z^{1 / \nu}
$$

where $\Xi$ is $\operatorname{Exp}(\lambda)$-distributed and $Z$ is Cauchy-distributed.

The behaviour of the fractional Dirichlet distribution in the case $N=2$ is shown in Fig. 2 for $\nu=0.7, \beta_{1}=2, \beta_{2}=3$. In this case, the generalized Dirichlet distribution is not a good approximation.

This is not the case for $N=2, \nu=0.7, \beta_{1}=0.2$ and $\beta_{2}=0.4$ where the generalized Dirichlet distribution is a reasonably good approximation of the fractional Dirichlet distribution. This is represented in Fig. 3 ,

For larger values of the parameters $\beta_{i}$, one gets a unimodal distribution in both cases as shown in Fig. 4 for $N=2, \nu=0.95, \beta_{1}=10, \beta_{2}=30$.

In Fig. 5 , the heavy character of the right tail of the generalized Dirichlet distribution is highlighted by the log-log plot.

\section{Acknowledgements}

F. Polito has been partially supported by the project "Memory in Evolving Graphs" (Compagnia di San Paolo/Università degli Studi di Torino).

\section{References}

[1] L. Beghin, E. Orsingher, Fractional Poisson processes and related planar random motions. Electron. J. Probab. 14, No 61 (2009), 1790-1827.

[2] J. Bertoin, Exchangeable Coalescents. ETH Zurich, 2010.

[3] L. Bondesson, A general result on infinite divisibility. Ann. Probab. 7, No 6 (1979), 965-979.

[4] D. O. Cahoy, F. Polito, Renewal processes based on generalized MittagLeffler waiting times. Comm. Nonlinear Sci. Numer. Simulat. 18, No 3 (2013), 639-650. 


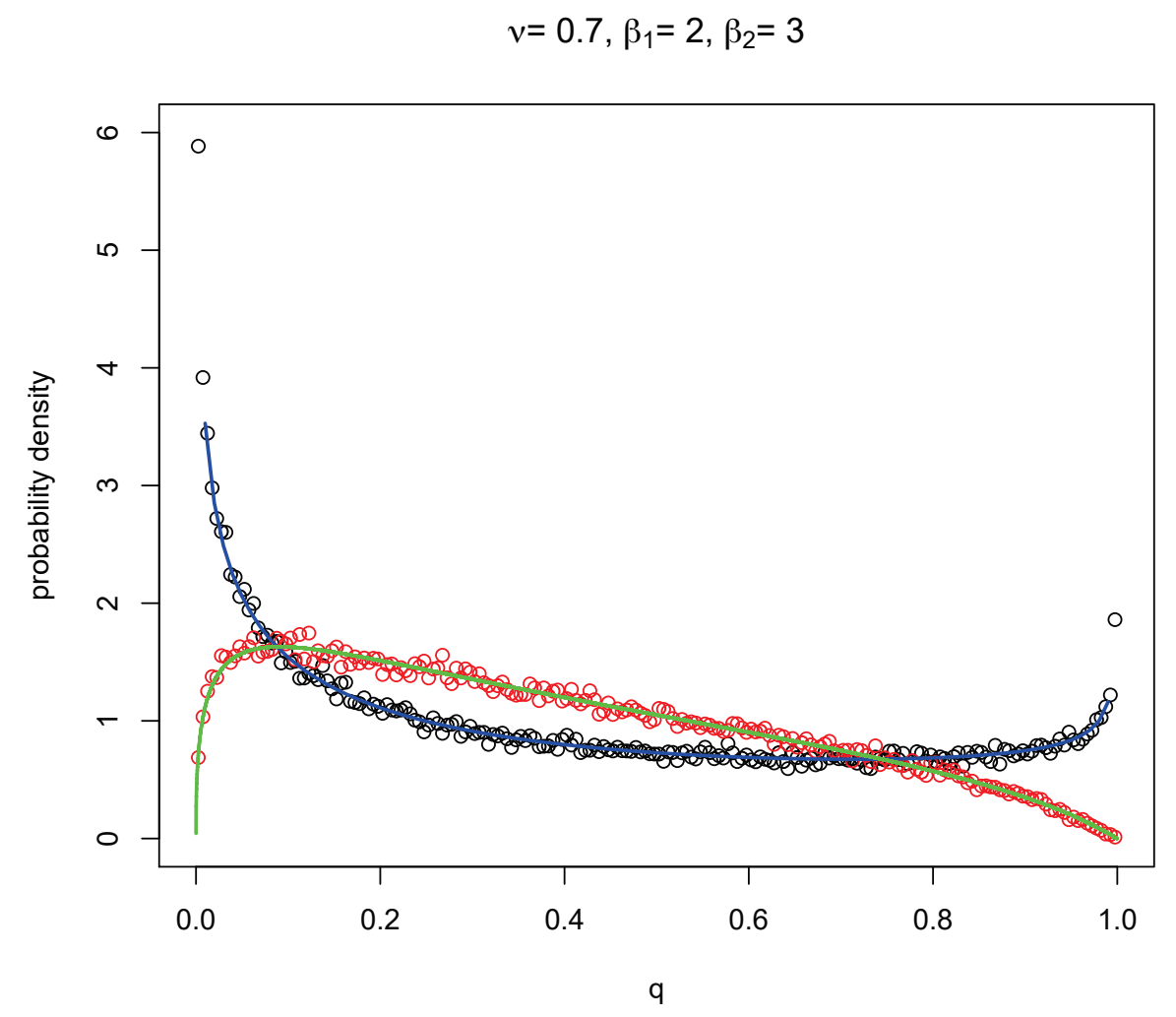

Figure 2. Pdf's for $\nu=0.7, \beta_{1}=2, \beta_{2}=3$. The black circles represent the results of a Monte Carlo simulation for the fractional Dirichlet distribution and the blue line is the corresponding theoretical value from equation (2.5). The red circles come from a Monte Carlo simulation of the generalized Dirichlet distribution and the green curve is the plot of the theoretical pdf.

[5] S. Favaro, G. Hadjicharalambous, I. Prünster, On a class of distributions on the simplex. J. Statist. Plann. Inference 141, No 9 (2011), 2987-3004.

[6] D. Fulger, E. Scalas, G. Germano, Monte Carlo simulation of uncoupled continuous-time random walks yielding a stochastic solution of the space-time fractional diffusion equation. Phys. Rev. E 77 (2008), Art. 021122 . 
A FRACTIONAL GENERALIZATION OF THE DIRICHLET... 133

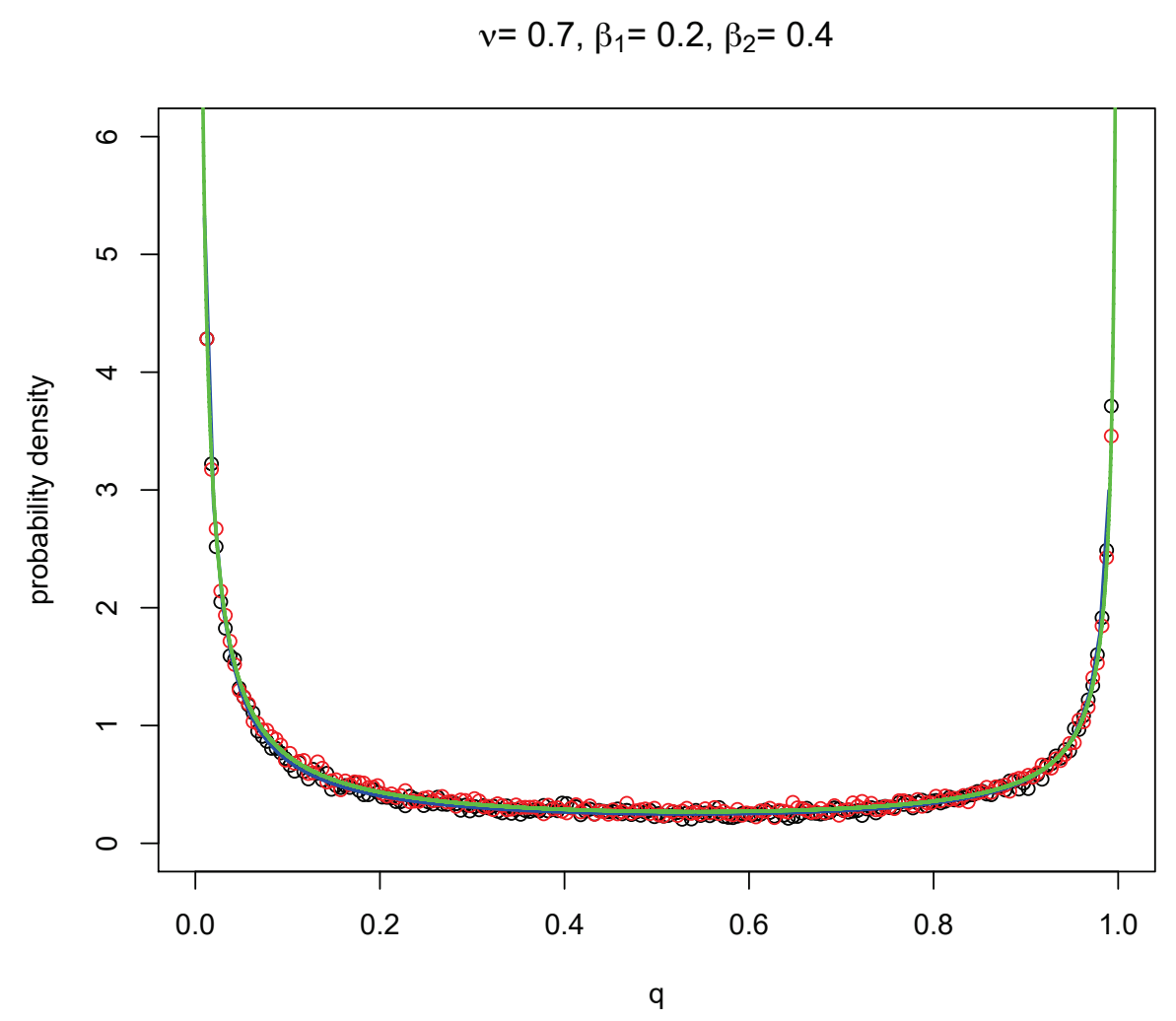

Figure 3. Pdf's for $\nu=0.7, \beta_{1}=0.2, \beta_{2}=0.4$. Circles and curves have the same meaning as in Fig. 2 ,

[7] A. Giusti, I. Colombaro, R. Garra, R. Garrappa, F. Polito, M. Popolizio, F. Mainardi, A practical guide to Prabhakar fractional calculus. Fract. Calc. Appl. Anal. 23, No 1 (2020), 9-54; DOI:10.1515/fca2020-0002; https://www.degruyter.com/view/journals/fca/23/1/

fca.23.issue-1.xml.

[8] R. Gorenflo, F. Mainardi, Fractional Calculus: Integral and Differential Equations of Fractional Order. In: Fractals and Fractional Calculus in Continuum Mechanics, A. Carpinteri and F. Mainardi (Eds.), Springer, New York and Wien (1997), 223-276.

[9] I. S. Gradshteyn, I. M. Ryzhik, Table of Integrals, Series, and Products. 8th Edition, Elsevier/Academic Press, Amsterdam (2015).

[10] R. D. Gupta, D. S. P. Richards, Multivariate Liouville distributions. J. Multivariate Anal. 23, No 2 (1987), 233-256. 


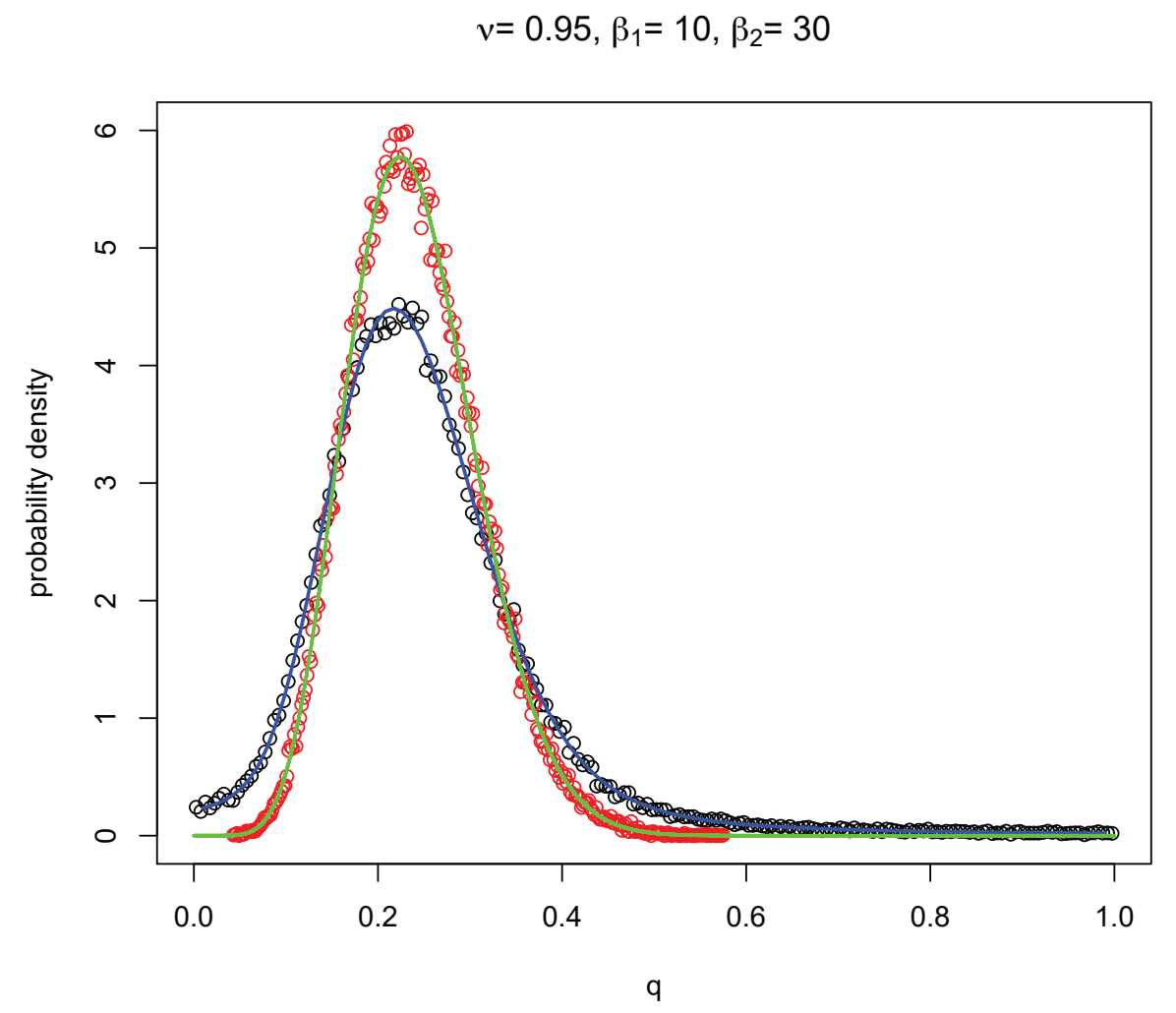

Figure 4. Pdf's for $\nu=0.95, \beta_{1}=10, \beta_{2}=30$. Circles and curves have the same meaning as in Fig. 2 .

[11] N. L. Johnson, S. Kotz, N. Balakrishnan, Continuous Univariate Distributions. Vol. 1. 2nd Edition, Wiley Ser. in Probability and Mathematical Statistics: Applied Probability and Statistics, John Wiley \& Sons, Inc., New York (1994).

[12] A. A. Kilbas, M. Saigo, H-Transforms. Theory and Applications. Ser. Analytical Methods and Special Functions, Vol. 9, Chapman \& Hall/CRC, Boca Raton, FL (2004).

[13] T. Kozubowski, Mixture representation of Linnik distributions revisited. Stat. Probab. Lett. 38 (1998), 157-160.

[14] F. Mainardi, R. Gorenflo, E. Scalas, A fractional generalization of the Poisson processes. Vietnam J. Math. 32 (Special Issue) (2004), 53-64.

[15] A. M. Mathai, H. J. Haubold, Special Functions for Applied Scientists. Springer, New York (2008). 
A FRACTIONAL GENERALIZATION OF THE DIRICHLET... 135

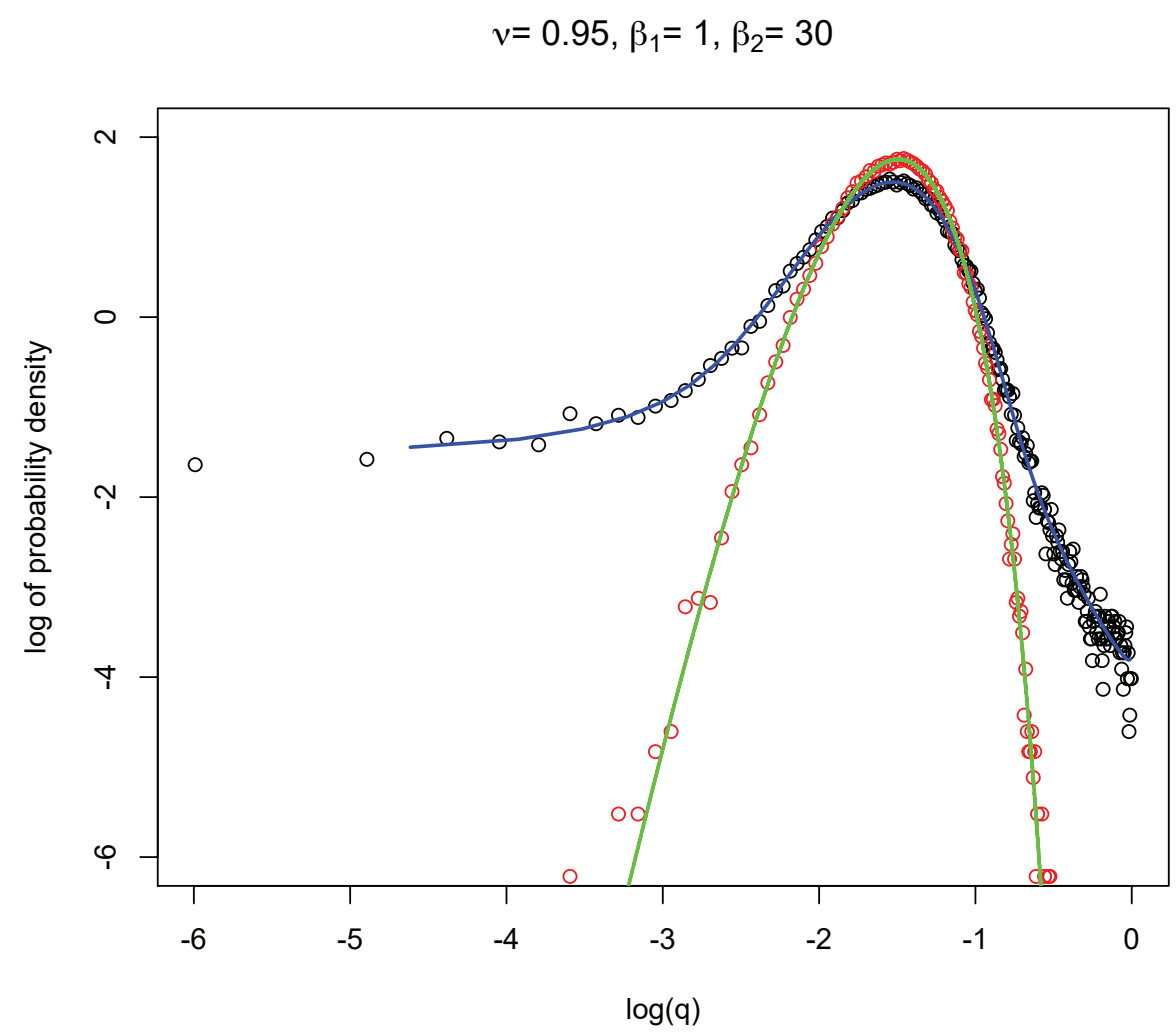

Figure 5. Double logarithmic plot of the pdf's for $\nu=$ 0.95, $\beta_{1}=10, \beta_{2}=30$. Circles and curves have the same meaning as in Fig. 2 ,

[16] T. M. Michelitsch, A. P. Riascos, Generalized fractional Poisson process and related stochastic dynamics. Fract. Calc. Appl. Anal. 23, No 3 (2020), 656-693; DOI:10.1515/fca-2020-0034; https://www.degruyter.com/view/journals/fca/23/3/

fca.23.issue-3.xml.

[17] T. M. Michelitsch, A. P. Riascos, Continuous time random walk and diffusion with generalized fractional Poisson process. Phys. A $\mathbf{5 4 5}$ (2020), Art. 123294.

[18] T. M. Michelitsch, F. Polito, A. P. Riascos, Biased continuous-time random walks with Mittag-Leffler jumps. Fractal and Fractional 4, No 4 (2020), Art. 51.

[19] R. N. Pillai, On Mittag-Leffler functions and related distributions. Ann. Inst. Statist. Math. 42, No 1 (1990), 157-161. 
${ }^{1}$ Department of Mathematics G. Peano

Università degli Studi di Torino

Via C. Alberto 10, 10123, Torino, ITALY

e-mails: elvira.dinardo@unito.it,federico.polito@unito.it

2 Department of Mathematics

University of Sussex

Falmer, Brighton, BN1 9QH, UK

e-mail: e.scalas@sussex.ac.uk (Corresponding author)

Received: November 8, 2020

Please cite to this paper as published in:

Fract. Calc. Appl. Anal., Vol. 24, No 1 (2021), pp. 112-136,

DOI: 10.1515 /fca-2021-0006 